\title{
Probing the relative orientation of molecules bound to DNA through controlled interference using second-harmonic generation
}

\author{
Benjamin Doughty, Yi Rao, Samuel W. Kazer, Sheldon J. J. Kwok, Nicholas J. Turro ${ }^{1}$, and Kenneth B. Eisenthal ${ }^{2}$ \\ Department of Chemistry, Columbia University, New York, NY 10027 \\ Contributed by Kenneth B. Eisenthal, February 7, 2013 (sent for review January 12, 2013)
}

\begin{abstract}
A method is described in which the interference of radiated secondharmonic electric fields generated by a pair of oriented molecules intercalated into double-stranded DNA is controlled and measured. The results show that the relative molecular orientation of the two molecules significantly changes the magnitude of the observed second-harmonic generation intensity, which is described by a simple model that accounts for the interferences of the radiated fields. The technique presented shows promise for future experiments investigating structural changes induced by the formation of a DNAbiomolecule complex.
\end{abstract}

alignment | daunorubicin | equilibrium binding | nonlinear spectroscopy | phase

Experiments are presented in which the relative spatial orienEtation of two molecules bound to a DNA duplex is systematically manipulated and subsequently probed using second-harmonic (SH) generation (SHG). Specifically, one daunomycin molecule was rotated relative to another by changing the number of base pairs separating them on a given DNA duplex that was affixed to a colloidal microparticle. Each DNA base pair on translation up or down the helix to the location of the adjacent base pair imparts a $36^{\circ}$ rotation to the helical DNA structure (1); thus, the relative orientation of DNA intercalators with defined recognition sites can be controlled by simply changing the number of bases pairs separating them on a given DNA duplex, as sketched in Fig. 1. The interference of $\mathrm{SH}$ fields generated from the intercalated species is exploited to map the measured $\mathrm{SH}$ intensity to the intercalated molecules' relative orientation. The interference of radiated $\mathrm{SH}$ fields generated from bound daunomycin molecules on a DNA duplex was measured and found to be modulated by the relative spatial orientation of the two drugs. The interference of the $\mathrm{SH}$ radiation was described in a simple model discussed below. Taking advantage of the tailored interference, the SHG method presented has the potential to serve as a solution phase "molecular protractor" to measure DNA unwinding angles for a broad range of protein-DNA and drug-DNA complexes.

SHG is a coherent surface specific spectroscopy where intense light at frequency $\omega$ induces a second-order polarization that oscillates at twice the driving laser frequency, and thereby generates light at twice the fundamental frequency, $2 \omega(2,3)$. Because SHG is coherent, the radiated $\mathrm{SH}$ intensity from the $k$ th particle in solution is given by the following:

$$
I_{2 \omega, k} \propto\left|\sum_{i} E_{2 \omega}^{i}\right|^{2}
$$

where $E_{2 \omega}^{i}$ is the SH field from a given interfacial molecule, which add up, with its respective phase, with other interfacial molecules to produce the overall $\mathrm{SH}$ intensity from an individual particle. The total SHG intensity measured in experiments is the incoherent sum of the coherent SH light generated by the daunomycin pair bound to the DNA on the individual particles in solution. The isotropic bulk medium does not produce coherent
$\mathrm{SH}$ radiation because the $\mathrm{SH}$ field generated by one randomly oriented molecule is, in principle, canceled out by another molecule that has the opposite orientation, leading to an overall destructive interference of the bulk SH signal; although incoherent $\mathrm{SH}$ light is generated in the bulk medium by density and orientational fluctuations of the species in bulk solution (hyper-Rayleigh scattering). Experiments have previously found that bulk water is responsible for the incoherent $\mathrm{SH}$ background signals (4). In the work reported here, the observed $\mathrm{SH}$ intensity generated by a pair of molecules bound to DNA, whose orientations are well defined and controlled, is shown to contain information on the relative orientation of the two molecules, allowing for detailed solution phase investigations of relative molecular orientation.

\section{Experimental}

The experimental apparatus has been described previously $(4,5)$. Briefly, a KM Labs Ti:sapphire oscillator running at $80 \mathrm{MHz}$, center wavelength of $840 \mathrm{~nm}$, producing $300 \mathrm{~mW}$ of average power with a pulse width of $\sim 60 \mathrm{fs}$ was focused into a 2-mm cuvette. The generated $\mathrm{SH}$ radiation was collected with a lens, telescoped, and filtered to remove much of the fundamental laser frequency before focusing into an Acton 300i spectrograph coupled to a Princeton Instruments Spec-10 CCD camera. The exposure was set to $1 \mathrm{~s}$; several hundred exposures per titration step were collected to obtain statistics. Each experiment was carried out in triplicate to ensure reproducibility and reduce uncertainties. All quoted uncertainties are given at the $95 \%$ confidence interval.

Biotinylated DNA (Integrated DNA Technologies) was coupled to $1-\mu \mathrm{m}$ NeutrAvidin-coated silica amine beads according to previously published procedures (5-7). In all experiments, the total concentration of DNA was 420 $\mathrm{nM}$, to ensure complete surface coverage; the particle density was $2.5 \times 10^{8}$ particles per $\mathrm{mL}$, and the samples were suspended in $50 \mathrm{mM}$ Tris buffer at $\mathrm{pH}$ 7.5. The $\mathrm{SH}$ intensity measured from the beads and excess DNA in solution (i.e., not attached to the particle) was the same as neat water, within the experimental uncertainty. Hyper-Rayleigh scattering from bulk water was found to be responsible for the background SH signal, which was subtracted from the measured SHG intensity in the data shown below. Excess DNA in solution was previously found to not influence the binding isotherms (4).

\section{Results and Discussion}

Daunomycin is an anticancer drug that is commonly used to treat acute leukemia (8-10), and has a somewhat unique property among small-molecule DNA binding agents in that it preferentially binds to triplet sequences in double-stranded DNA (1014). The work here makes use of the specificity of daunomycin to the sequence TCG to spatially orient the two intercalated drugs on a given DNA duplex. Daunomycin has the effect of lengthening the DNA helical axis by $\sim 3.4 \AA$ and imparting a small unwinding of the DNA helix $\left(\sim 8^{\circ}\right)(15-17)$ relative to noncomplexed B-DNA. The sequence of the DNA 33-mer duplex that was used in the experiments was referenced to the sequence

Author contributions: B.D., Y.R., S.W.K., S.J.J.K., N.J.T., and K.B.E. designed research; B.D. Y.R., S.W.K., S.J.J.K., N.J.T., and K.B.E. performed research; B.D., Y.R., S.W.K., S.J.J.K., N.J.T. and K.B.E. analyzed data; and B.D., Y.R., S.W.K., S.J.J.K., N.J.T., and K.B.E. wrote the paper The authors declare no conflict of interest.

${ }^{1}$ Deceased November 24, 2012

${ }^{2}$ To whom correspondence should be addressed. E-mail: kbe1@columbia.edu. 


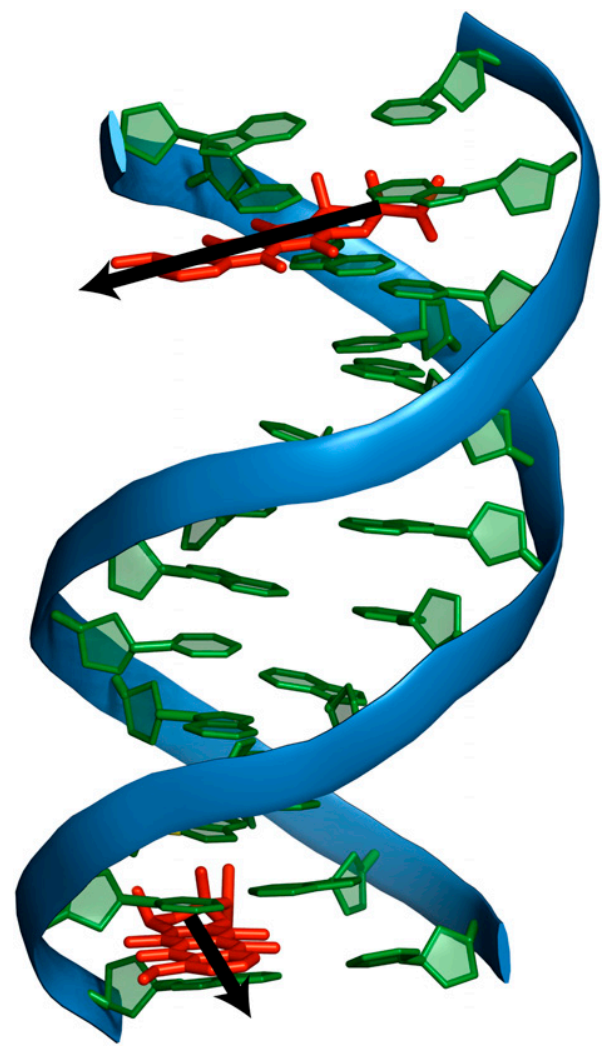

Fig. 1. The relative orientation of two daunomycin molecules (orange with black arrows) bound to DNA is sketched here (omitting the amino sugar for clarity).

used where only one recognition site was present: 5'-CTC AAG TGA ACT CAA GTG AAT CCA ATC GAA GTT-3'; the recognition site is highlighted and the complementary strand is implied for succinctness. To change the orientation angle between the two drugs on a DNA duplex, the distance between two recognition sites was varied by changing the number of base pairs separating the two recognition sites. For instance, the DNA used where the spacing was 20 bases apart was $5^{\prime}$-CTC AAT CGA ACT CAA GTG AAT CCA ATC GAA GTT-3'.

Representative binding isotherms collected in the experiment are shown in Fig. 2. It was observed that the $\mathrm{SH}$ intensity at the maximum density of bound daunomycin, which is the plateau of the isotherm, changed as the spacing between the two recognition sequences was varied. The orientation angle between the two daunomycin molecules is given by $\Phi=n * 36^{\circ}$, where $n$ is the number of base pairs separating the two recognition sites. Therefore, when $n=5,15,25$, etc., the two binding sites are antiparallel and the SHG will destructively interfere; similarly, when $n=10,20,30$, etc., the intercalated drugs are aligned parallel and will constructively interfere, as observed from the $\mathrm{SH}$ binding data in Fig. 2.

To quantify the modulated SHG intensity, the signal intensity at the isotherm plateau was averaged and plotted vs. number of base pairs separating the two binding sites in Fig. 3. This plot more clearly illustrates the oscillatory behavior that was expected to occur by systematically rotating one daunomycin relative to another by changing the number of base pairs separating them, and collecting the resulting SH light. The relative orientation is depicted in Fig. 3 as arrows representing the individual drug molecules.

A simple model was implemented to quantify the interference of radiated $\mathrm{SH}$ fields from the daunomycin molecules on a DNA duplex, which is given below. A coordinate system is defined according to Fig. 4 where two generated SH fields, $\boldsymbol{E}_{\boldsymbol{a}}$ and $\boldsymbol{E}_{\boldsymbol{b}}$ are defined relative to the $x$ axis by the angles $\phi_{a}$ and $\phi_{b}$ for $\boldsymbol{E}_{\boldsymbol{a}}$ and $\boldsymbol{E}_{\boldsymbol{b}}$, respectively (bold type indicates vector quantities). Similarly, the angle out of the $x-y$ plane is defined as $\theta_{a}$ and $\theta_{b}$. The SH intensity due to the intercalated daunomycin molecules, $a$ and $b$, is the coherent sum of their individual SH fields and is written as follows:

$$
I_{2 \omega} \propto\left|\boldsymbol{E}_{\boldsymbol{a}}+\boldsymbol{E}_{\boldsymbol{b}}\right|^{2}=\left|\boldsymbol{E}_{\boldsymbol{a}}\right|^{2}+\left|\boldsymbol{E}_{\boldsymbol{b}}\right|^{2}+2 \boldsymbol{E}_{\boldsymbol{a}} \cdot \boldsymbol{E}_{\boldsymbol{b}}
$$

where each $\mathrm{SH}$ field can be written explicitly in terms of vector components to give the following:

$$
\boldsymbol{E}_{\boldsymbol{a}}=E_{a}\left(\sin \theta_{a} \cos \phi_{a} \hat{\boldsymbol{x}}+\sin \theta_{a} \sin \phi_{a} \hat{\boldsymbol{y}}+\cos \phi_{a} \hat{z}\right) .
$$

The DNA's helical axis is defined along the $z$ axis in this coordinate system so the DNA bases lie parallel to the $x-y$ plane. Based on the crystal structure of the daunomycin-DNA complex, daunomycin is known to intercalate parallel to the DNA's bases (15-17) yielding the approximation, $\theta_{a} \sim \theta_{b} \sim 90^{\circ}$. Applying this approximation to Eq. 3 and substituting into Eq. 2 yields the following:

$$
I_{2 \omega} \propto\left|\boldsymbol{E}_{\boldsymbol{a}}\right|^{2}+\left|\boldsymbol{E}_{\boldsymbol{b}}\right|^{2}+2\left|\boldsymbol{E}_{\boldsymbol{a}}\right|\left|\boldsymbol{E}_{\boldsymbol{b}}\right|\left(\cos \phi_{a} \cos \phi_{b}+\sin \phi_{a} \sin \phi_{b}\right) .
$$

Simplifying and noting that the intensity is proportional to the orientational average of the intercalated daunomycin-DNA complex on a given particle gives the following:

$$
I_{2 \omega} \propto\left|\boldsymbol{E}_{\boldsymbol{a}}\right|^{2}+\left|\boldsymbol{E}_{\boldsymbol{b}}\right|^{2}+2\left|\boldsymbol{E}_{\boldsymbol{a}}\right|\left|\boldsymbol{E}_{\boldsymbol{b}}\right|\left\langle\cos \left(\phi_{a}-\phi_{b}\right)\right\rangle .
$$

Setting $\Phi=\phi_{a}-\phi_{b}$ and noting that $\left|\boldsymbol{E}_{\boldsymbol{a}}\right|=\left|\boldsymbol{E}_{\boldsymbol{b}}\right| \equiv\left|E_{2 \omega}\right|$ yields the following:

$$
I_{2 \omega} \propto 2\left|E_{2 \omega}\right|^{2}[1+\langle\cos \Phi\rangle]
$$

where $\left|E_{2 \omega}\right|^{2}$ represents the generated SH intensity of one daunomycin molecule bound to DNA. The SHG signal from a single silica particle is the coherent summation of all of the individual daunomycin-DNA complexes attached to the particle. The total SHG signal is the incoherent sum of the SHG from the individual particles. The orientational average in Eq. 6 contains the variation in daunomycin orientations (e.g., tilt angles, etc.) relative to the DNA helical axis. To fit the

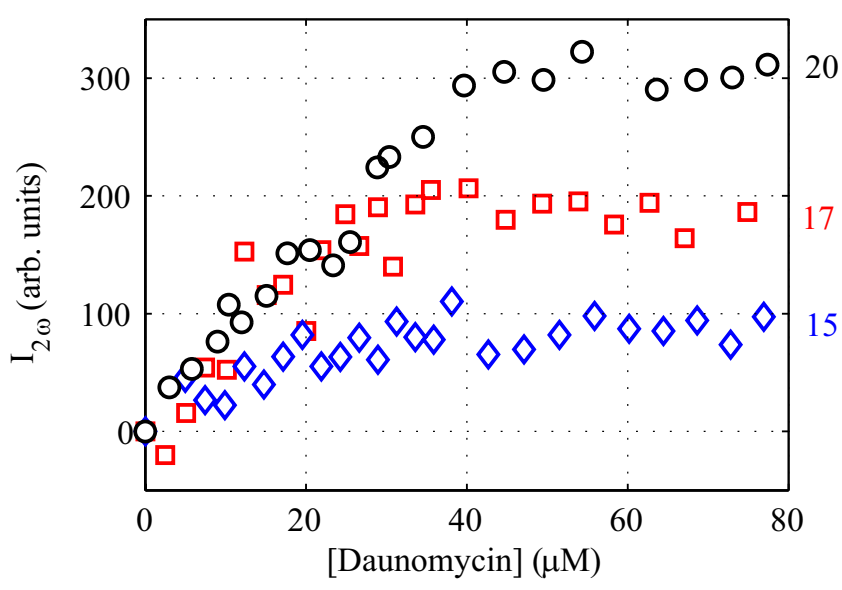

Fig. 2. Representative binding isotherms collected with different numbers of base pairs separating two daunomycin molecules bound to DNA (listed on Right). 


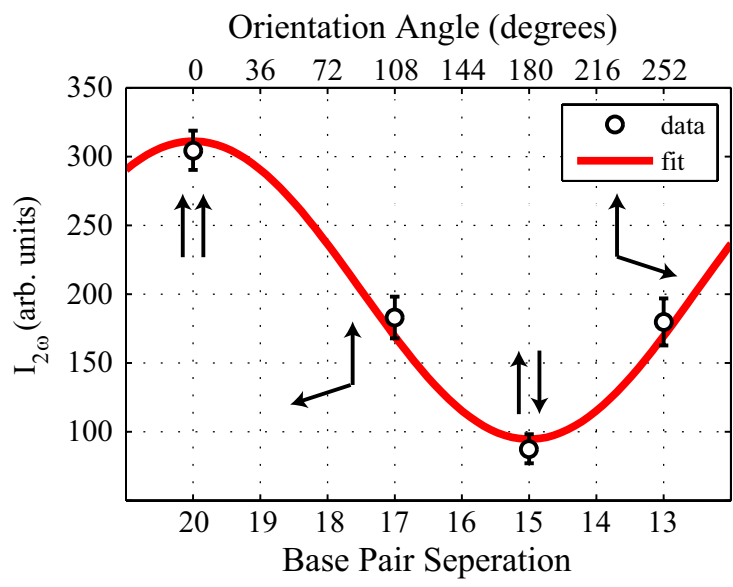

Fig. 3. The SH intensity is plotted vs. base pair separation on the lower axis, and orientation angle on the upper axis. The relative orientation of the bound daunomycin molecules is sketched as arrows that are associated with each data point.

experimental data, the orientational average above is replaced with an empirical parameter, $g$, and summing over the particles yields the following:

$$
I_{2 \omega}(\text { total }) \propto \sum_{k} I_{2 \omega, k}=2[1+g \cdot \cos \Phi] \sum_{k}\left|E_{2 \omega, k}\right|^{2} .
$$

The experimental data in Fig. 3 was fit to Eq. 7 to yield $g=$ $0.53 \pm 0.17$ and $\sum_{k}\left|E_{2 \omega, k}\right|^{2}=101 \pm 15$ arbitrary units (arb. units). The value of $\sum_{k}\left|E_{2 \omega, k}\right|^{2}$ extracted from the fit is in agreement with the $\mathrm{SH}$ intensity that was observed in experiments with one recognition site $(120 \pm 17$ arb. units $)$, as expected. The agreement between the fit and the measured intensity from the binding of daunomycin to one recognition site serves as an internal consistency check to ensure the model and fit retrieve meaningful parameters.

The retrieved value of $g$ indicates that the interference "efficiency" is $\sim 53 \%$. The deviation from ideal $\mathrm{SH}$ interference might be due to the ensemble distribution of orientations that the two drugs can take relative to one another, resulting in altered relative orientations, e.g., the daunomycin molecules might be twisted or tilted slightly from one another when intercalated into the DNA. Imperfect interference could also occur from structural and orientational fluctuations occurring on timescales much faster than the measurement. The slight unwinding of DNA by daunomycin could affect the interference of the SHG

1. Bloomfield VA, Crothers DM, Tinoco I (2012) Nucleic Acids: Structures, Properties, and Functions (University Science Books, Sausalito, CA).

2. Eisenthal KB (2006) Second harmonic spectroscopy of aqueous nano- and microparticle interfaces. Chem Rev 106(4):1462-1477.

3. Shen YR (2003) Principles of Nonlinear Optics (Wiley Interscience, New York).

4. Doughty B, et al. (2013) Binding of the anti-cancer drug daunomycin to DNA probed by second harmonic generation. J Phys Chem B, 10.1021/jp311634a.

5. Doughty B, Kazer SW, Eisenthal KB (2011) Binding and cleavage of DNA with the restriction enzyme EcoR1 using time-resolved second harmonic generation. Proc Nat/ Acad Sci USA 108(50):19979-19984.

6. Leunissen ME, et al. (2009) Switchable self-protected attractions in DNA-functionalized colloids. Nat Mater 8(7):590-595.

7. Leunissen ME, Dreyfus R, Sha R, Seeman NC, Chaikin PM (2010) Quantitative study of the association thermodynamics and kinetics of DNA-coated particles for different functionalization schemes. J Am Chem Soc 132(6):1903-1913.

8. Tan C, Tasaka H, Yu K-P, Murphy ML, Karnofsky DA (1967) Daunomycin, an antitumor antibiotic, in the treatment of neoplastic disease. Clinical evaluation with special reference to childhood leukemia. Cancer 20(3):333-353.

9. Tse WC, Boger DL (2004) Sequence-selective DNA recognition: natural products and nature's lessons. Chem Biol 11(12):1607-1617.

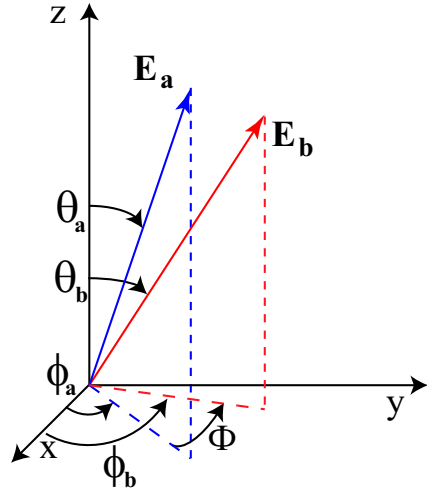

Fig. 4. Depiction of the relative angles between $\mathrm{SH}$ fields generated by the $a$ and $b$ daunomycin molecules bound to DNA.

fields, which would be observed in Fig. 3 as a phase shift (i.e., the maxima and minima would not coincide with $0^{\circ}$ or $180^{\circ}$, respectively). Because the data are well described by Eq. 7 (Fig. 3), it is surmised that daunomycin-induced changes in the DNA structure are within the experimental error of the results reported here. Despite its simplicity, the model successfully recovered the correct oscillation frequency of the SH intensity vs. base pair separation.

\section{Conclusions}

The results presented here demonstrate that the interference of second-harmonic fields generated by a pair of molecules complexed to double-stranded DNA can be used to probe their relative orientation. The straightforwardness of the experimental approach to control and measure relative molecular orientation, e.g., the anticancer drug daunomycin complexed with DNA investigated in this study, presents opportunities to probe structural aspects of biomolecular complexes in solution. For instance, it could be possible to investigate structural changes in DNA due to complex formation with proteins or drugs, e.g., one would measure a phase shift in the SH intensity vs. the number of base pairs separating the intercalated daunomycin molecules, and thus, their relative orientation angle would differ before and after complex formation of DNA with the ligand of interest.

ACKNOWLEDGMENTS. We acknowledge many useful conversations with Dr. Joseph R. Roscioli and Dr. Louis H. Haber. K.B.E. acknowledges generous funding from National Science Foundation (NSF) Eager Award CHE-1041980 NSF Grant CHE-1057483, and Defense Threat Reduction Agency Grant HDTRA1-11-1-0002. N.J.T. received funding from NSF Grants CHE-11-11398 and DRM 02-13774.

10. Chaires JB (1996) Molecular recognition of DNA by daunorubicin. Advances in DNA Sequence Specific Agents, ed Chaires JB (JAI, London), Vol 2, pp 141-167.

11. Chaires JB, Fox KR, Herrera JE, Britt M, Waring MJ (1987) Site and sequence specificity of the daunomycin-DNA interaction. Biochemistry 26(25):8227-8236.

12. Chaires JB, Herrera JE, Waring MJ (1990) Preferential binding of daunomycin to $5^{\prime}$ ATCG and 5'ATGC sequences revealed by footprinting titration experiments. Biochemistry 29(26):6145-6153.

13. Moore MH, Hunter WN, d'Estaintot BL, Kennard O (1989) DNA-drug interactions. The crystal structure of d(CGATCG) complexed with daunomycin. J Mol Biol 206(4): 693-705.

14. Chaires JB (1990) Biophysical chemistry of the daunomycin-DNA interaction. Biophys Chem 35(2-3):191-202.

15. Frederick CA, et al. (1990) Structural comparison of anticancer drug-DNA complexes: Adriamycin and daunomycin. Biochemistry 29(10):2538-2549.

16. Quigley GJ, et al. (1980) Molecular-structure of an anti-cancer drug-DNA complexdaunomycin plus d(CPGPTPAPCPG). Proc Natl Acad Sci USA 77(12):7204-7208.

17. Wang AHJ, Ughetto G, Quigley GJ, Rich A (1987) Interactions between an anthracycline antibiotic and DNA: molecular structure of daunomycin complexed to d(CpGpTpApCpG) at 1.2-A resolution. Biochemistry 26(4):1152-1163. 